\title{
Fontes auxiliares reguladas/isoladas/sincronizadas para aplicação em UPS
}

\section{Regulated/isolated/synchronized auxiliary sources for UPS applications}

\author{
Kleber dos Santos Lopes ${ }^{1}$; Carlos Henrique Gonçalves Treviso²; Rodolfo Barrteto \\ Canônico ${ }^{3}$
}

\section{Resumo}

Tendo em vista o panorama atual onde a expansão do consumo de energia elétrica possui um crescimento vigoroso, alavancando a quantidade de aplicações que não podem sofrer com a falta da mesma e aumentando-se assim a quantidade de sistemas ininterruptos de energia (UPS), este trabalho mostrará o projeto completo de um sistema de fontes para utilização em sistemas UPS. Desta forma, foi implementado um sistema com nove fontes reguladas, isoladas, limitadas em corrente e que podem ser sincronizadas por meio de um sinal externo ou auto sincronizadas, além disto, o mesmo apresenta grande vantagem em relação às tecnologias lineares utilizadas atualmente no que diz respeito a tamanho, peso, volume, flexibilidade e confiabilidade.

Palavras-chave: UPS. fontes chaveadas. fontes isoladas. sincronismo. limitação de corrente.

\begin{abstract}
Considering the current situation where the expansion of energy consumption has a vigorous growth, leveraging the amount of applications that can not suffer with the lack of it and thus increasing the amount of uninterruptible power systems (UPS), this paper will show the complete design of a font system for use in UPS. Thus, was implemented a system with nine regulated, isolated, current limited power sources and that can be synchronized at an external signal or synchronized self, moreover, it presents a great advantage over linear technologies in terms respect to size, weight, volume, flexibility and reliability.
\end{abstract}

Key words: UPS. switching supplies. isolated supplies. synchronism. current limiting.

\section{Introdução}

A demanda por energia elétrica vem crescendo anualmente e juntamente com este crescimento estão aumentando as aplicações que não podem sofrer a falta da mesma, como por exemplo, hospitais, aeroportos, centros computacionais, centrais de telefonia, centrais de banco de dados e muitas outras, segundo (FERREIRA, 2009; TREVISO; DEMIAN JUNIOR; FERREIRA, 2009; CÂMARA; CRUZ; BASCOPÉ, 2010; SCHIAVON, 2007; LINARD et

\footnotetext{
${ }^{1}$ Aluno de mestrado do Departamento de Engenharia Elétrica, Universidade Estadual de Londrina; kleber@identech.com.br

${ }^{2}$ Docente do Departamento de Engenharia Elétrica da Universidade Estadual de Londrina - DEEL-UEL; treviso@uel.br

${ }^{3}$ Graduado e Mestre em Engenharia Elétrica, Universidade Estadual de Londrina
} 
al., 2008). Infelizmente a abrangência e a qualidade do fornecimento de energia não acompanham este aumento. No Brasil somente no primeiro semestre de 2010 foram pagas mais de 41,511 milhões de ações de compensações por problemas no fornecimento de energia segundo (AGÊNCIA NACIONAL DE ENERGIA ELÉTRICA, 2011).

Por este motivo, segundo (FERREIRA, 2009; TREVISO; DEMIAN JUNIOR; FERREIRA, 2009) a utilização de sistemas ininterruptos de energia ou UPS (também conhecidos no Brasil como NoBreak) vem sofrendo um aumento vigoroso.

Muitos trabalhos vêm sendo feitos e apresentados sobre os UPS, mas em boa parte deles, são apresentadas apenas a parte de controle e potência, deixando a geração das fontes necessárias para os mesmos em segundo plano ou até mesmo fora do escopo.

Os UPS, assim como muitos outros circuitos de geração/transformação de energia necessitam de várias fontes em referências distintas para o seu funcionamento. Segundo (FERREIRA, 2009) apenas para um inversor monofásico (na topologia ponte completa) são necessárias três fontes com referências distintas e segundo (CANÔNICO, 2011) se forem utilizadas técnicas que trabalham com tensões negativas no desacionamento das chaves, passam a ser necessárias quatro fontes com referências distintas (considerando que umas das referências é a mesma do circuito de controle).

A Figura 1 mostra o esquema típico de um inversor monofásico na topologia ponte completa (KASTER; PAGANO, 2006), onde as chaves S1, $\mathrm{S} 2$, S3 e S4 devem ser acionadas através de drivers, que para executarem esta função necessitam de fontes em referências distintas.

Ainda se fazem necessárias fontes isoladas para o controle do circuito elevador de tensão, e para o circuito retificador de tensão.
Figura 1 - Esquema básico de um inversor.

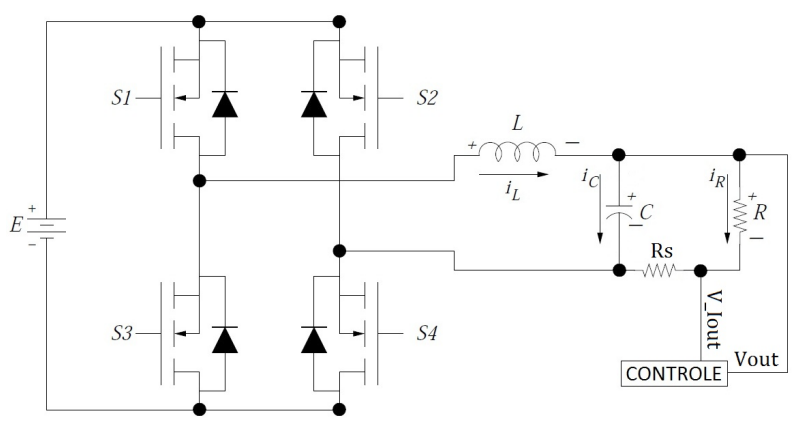

Fonte: KASTER; PAGANO, 2006 - adaptado pelo autor

Logo, se considerarmos a utilização de três fontes isoladas no circuito inversor (desconsiderando a fonte de mesmo referencial do circuito de controle), três fontes isoladas para o circuito elevador de tensão, e três fontes para o retificador de tensão, seriam necessárias nove fontes isoladas para o projeto de um UPS.

A Figura 2 mostra o diagrama de blocos de um sistema UPS genérico (TREVISO; SCHIAVON, 2007) que utiliza a topologia "ponte completa" em todos os conversores. Nesta figura foram feitas referências das fontes que seriam necessárias para alimentar o sistema, caso alguma técnica que utilize tensões negativas no desacionamento das chaves fosse utilizada (CANÔNICO, 2011).

Figura 2 - UPS genérico com referência as fontes de alimentação.

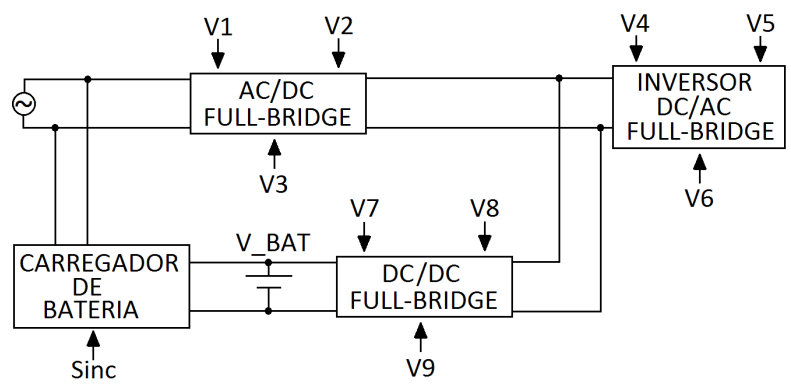

Fonte: TREVISO; SCHIAVON, 2007 - adaptado pelo autor 
Outro fator crucial para as fontes do UPS é o sincronismo entre as mesmas e o circuito de controle, o que segundo (SCHIAVON, 2007; CANÔNICO, 2011; MILNE et al., 1998), mantém os ruídos de chaveamento em uma faixa de freqüência fixa, reduzindo o número de harmônicas indesejadas e facilitando a filtragem.

É desejável também que estas fontes sejam reguladas, pois em alguns casos, os circuitos alimentados pelas mesmas utilizam circuitos integrados que possuem um valor mínimo de tensão de alimentação ou circuitos que utilizam divisores de tensão para comparações, assim, variações desta tensão podem ocasionar problemas no funcionamento destes circuitos o que pode gerar falhas graves, como por exemplo, o acionamento de duas chaves "concorrentes" ao mesmo tempo, gerando curtoscircuitos no sistema.

Como todo sistema é passível de sofrer falhas ou efeitos transitórios, é uma grande vantagem as fontes possuírem proteções contra sobre corrente, evitando assim a queima de parte dos componentes em situações críticas, como um curto-circuito.

Considerando ainda que a forma mais utilizada em UPS para o armazenamento de energia são as baterias (SCHUCH et al., 2004), é necessária a disponibilidade de um pulso de sincronismo isolado para a utilização no circuito carregador destas baterias, mantendo assim todas as partes do UPS em sincronismo.

Como em vários trabalhos publicados atualmente sobre sistemas UPS, pouco se fala sobre a forma como as fontes necessárias para o funcionamento do mesmo são geradas e quais características citadas anteriormente elas atendem, este trabalho visa sanar esta necessidade encontrada, trazendo o projeto completo de um sistema de nove fontes chaveadas, reguladas, isoladas, sincronizadas, com limitação de corrente, no menor tamanho, peso e volume possíveis.

Além de atender todas as características desejáveis para um UPS, o sistema foi desenvolvido também para ser o mais flexível possível. A topologia escolhida para a fonte principal permite sua fácil alteração para outros valores de tensão de entrada (maiores ou menores que o utilizado neste trabalho), as fontes podem ser sincronizadas através de uma onda quadrada externa ou se auto-sincronizar (permitindo o funcionamento autônomo), as fontes secundárias podem ser projetadas com metade ou um quarto da freqüência externa (e com $49 \%$ ou $98 \%$ de razão cíclica), o número de fontes isoladas pode ser alterado apenas adicionando ou retirando conjuntos de placas do sistema, a tensão e/ou corrente de saída podem ser facilmente alteradas apenas refazendo o projeto das fontes secundárias, a limitação da corrente de saída pode ser alterada através do ajuste de potenciômetros e pode ser diferente para cada saída.

\section{Sistema Proposto}

Na Figura 3 está apresentado um diagrama de blocos do sistema proposto, e no Anexo 1 estão apresentados todos os circuitos da fonte principal, da divisão e adequação do sinal de entrada, o circuito detector de sinal externo, um dos transformadores isoladores e todo o circuito de uma das fontes secundárias.

Afonteprincipalgera 15 Vestáveis, paraautilização do restante do circuito e um sinal de sincronismo que pode ser utilizado na ausência do sincronismo externo. Existe um bloco que divide a freqüência do sinal externo e gera ondas complementares a partir do mesmo. Estas ondas complementares são utilizadas em conjunto com os transformadores isoladores para gerar as tensões isoladas. Com as tensões já isoladas, fontes secundárias são utilizadas para estabilizar, e controlar a tensão de saída.

Como o projeto prevê nove fontes, optou-se por trabalhar com cinco transformadores isoladores, sendo o quinto também com dois secundários, assim um dos secundários deste transformador foi utilizado para completar as nove fontes isoladas e o outro utilizado para disponibilizar o sinal de sincronismo isolado. 
Figura 3 - Diagrama de blocos do sistema proposto.

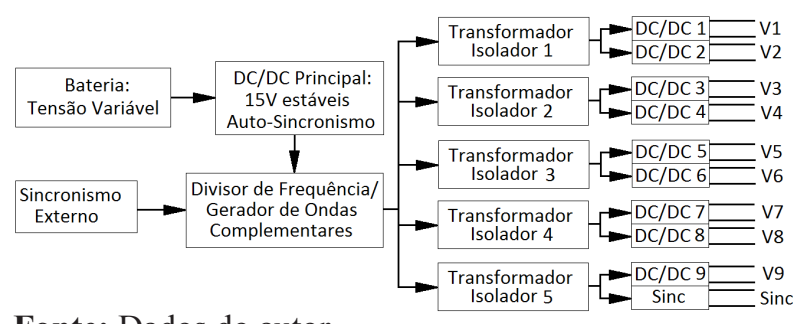

Fonte: Dados do autor

Para o cálculo dos transformadores isoladores e das fontes chaveadas foram utilizadas as equações de (MELLO, 1996).

O sistema pode trabalhar com sincronismo externo ou auto sincronizado, assim, utiliza-se um circuito para a detecção desta onda externa. Este circuito está apresentado na Figura 4. Como pode ser observado, existe uma porta inversora para adequar o sinal e através do capacitor $\mathrm{C} 1$ e dos resistores R4 e R5, o transistor Q2 estará saturado sempre que existirem pulsos na entrada, o que fará com que o transistor Q1 também sature indicando a presença do sinal externo.

Figura 4 - Circuito detector de sinal externo.

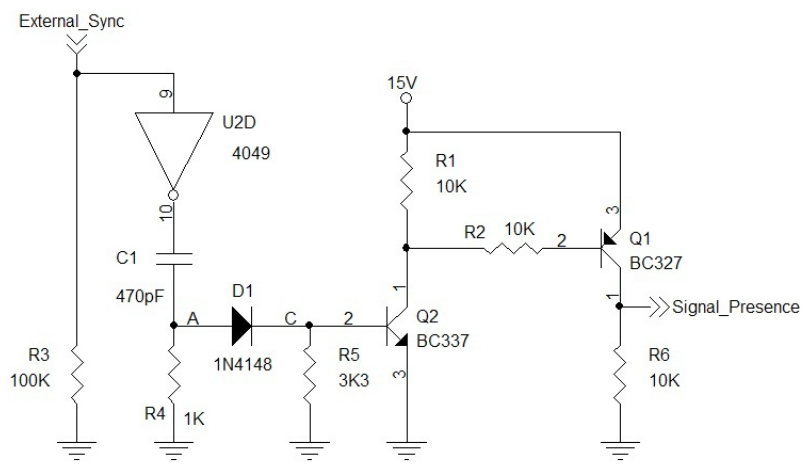

Fonte: Dados do autor

Para a geração dos pulsos de sincronismo, primeiramente é feito um tratamento no sinal de entrada através de um divisor de freqüências implementado com o flip-flop tipo D HEF4027, assim são gerados pulsos quadrados e complementares com metade ou um quarto da freqüência original. Quatro jumpers são utilizados na placa para selecionar a divisão de freqüência desejada, aumentando assim as possibilidades no projeto das fontes $\mathrm{CC} / \mathrm{CC}$ do sistema.

A topologia escolhida para a fonte principal foi "Flayback" no modo de funcionamento descontínuo. Esta topologia foi escolhida por permitir tensões de entrada maiores ou menores que a tensão desejada na saída (para este projeto foi escolhida tensão de entrada entre $8 \mathrm{~V}$ e $15 \mathrm{~V}$, considerando uma bateria, e tensão de saída de $15 \mathrm{~V}$, mas estes valores podem ser facilmente alterados caso utilize mais baterias em série). $\mathrm{O}$ modo de funcionamento descontínuo foi escolhido por apresentar núcleos menores do que seriam no modo contínuo. Outra vantagem da topologia "Flyback" é a possibilidade da limitação da corrente de saída bem como sua simplicidade.

O controle da fonte principal foi feito através do CI SG3525, que além de possuir toda a inteligência necessária embarcada, também permite facilmente o sincronismo através de um pino de entrada.

Quando o sinal externo não estiver presente, é utilizado o sinal de freqüência do oscilador "RC" do próprio SG3525 para gerar o pulso de autosincronismo das demais partes do sistema, como pode ser visto na Figura 5.

Figura 5 - Gerador de sincronismo a partir do oscilador "RC" do SG3525

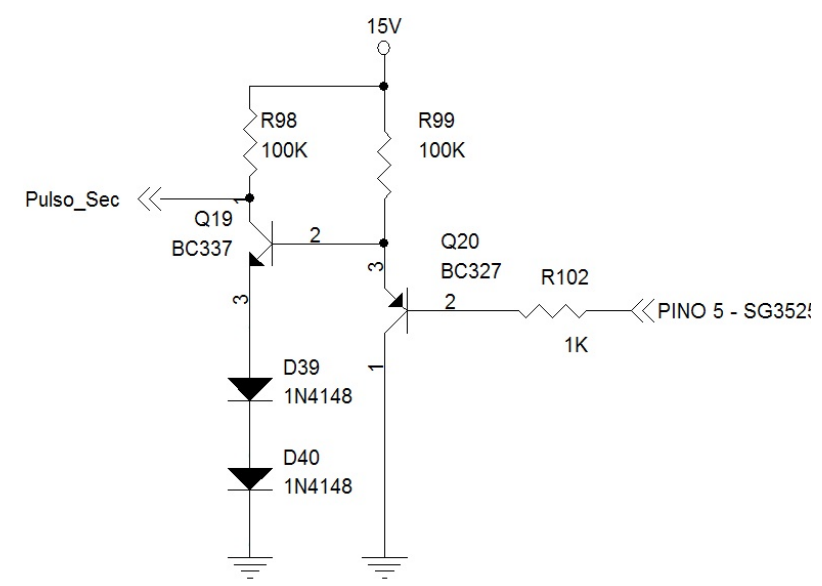

Fonte: Dados do autor 
Utilizou-se o CI HEF4066 para selecionar a origem do sincronismo, externo ou autosincronismo, para o restante do sistema.

Para isolar as fontes de saída, foram utilizados transformadores chaveados na freqüência determinada pela onda externa (no caso do protótipo montado a freqüência foi de $75 \mathrm{kHz}$ ). Visando a otimização dos núcleos utilizados para esta função, optou-se por fazer transformadores com tape central (melhorando o rendimento do núcleo) e dois secundários (diminuindo a quantidade de núcleos necessários).

Embora as saídas do flip-flop sejam complementares, o tempo de acionamento/ desacionamento dos transistores que chaveiam os transformadores isoladores faz com que os mesmos dissipem grandes quantidades de energia nos cruzamentos entre a saturação de um dos transistores e o corte do outro. Para minimizar este problema foram inseridos componentes em série com os pulsos de saída do flip-flop, para realizar atrasos neste sinal.

Como pode ser observado na Figura 6, o capacitor que está em paralelo com o gate do transistor é carregado mais lentamente, na subida do pulso, através do resistor que está em série com a saída do flip-flop. Mas na borda de descida, o capacitor é descarregado rapidamente através do diodo que está em paralelo com o resistor. Isto faz com que o transistor demore um pouco mais para entrar em saturação, mas rapidamente entre no estado de corte, diminuído a duração do cruzamento entre o corte do primeiro transistor e a saturação do segundo.
Figura 6 - Circuito de tratamento dos pulsos de acionamento dos transistores dos transformadores isoladores

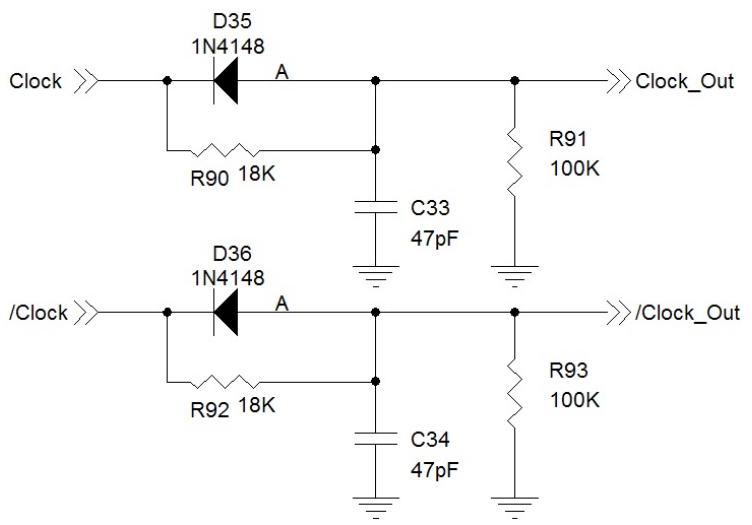

Fonte: Dados do autor

Como pode ser observado na Figura 7, o sincronismo das fontes secundárias foi obtido através do próprio sinal pulsado presente no secundário dos transformadores isoladores. Com o sinal de sincronismo sendo extraído nas duas bordas do sinal chaveado, é possível obter um sincronismo com o dobro da freqüência original.

Figura 7 - Aquisição do sinal de sincronismo isolado.

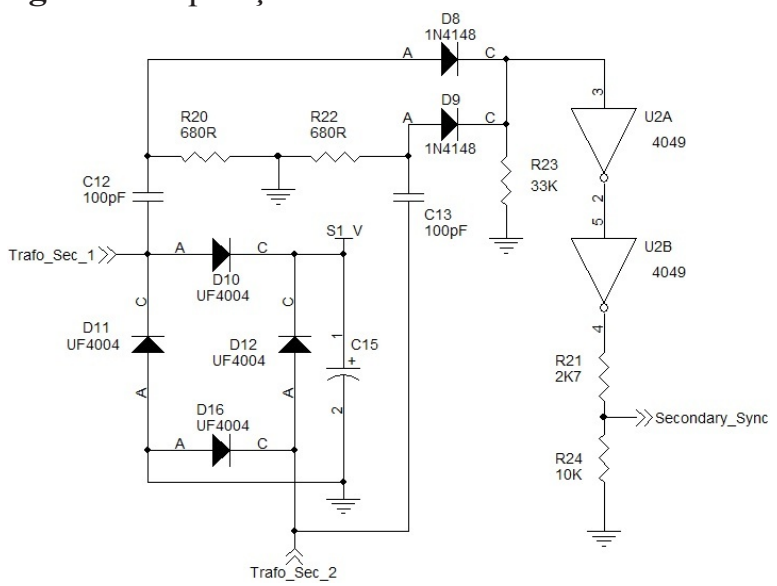

Fonte: Dados do autor 
Para aproveitar ao máximo a capacidade do circuito de controle, utilizou-se dois diodos para somar os pulsos de saída do SG3525, obtendo um sinal de controle com a mesma freqüência do sincronismo (150kHz no caso do protótipo montado) visto que cada saída é limitada a metade da freqüência de trabalho. Infelizmente o sinal resultante fica um pouco distorcido, assim, fez-se necessária a utilização de duas portas inversoras para sua readequação. Como ficam sobrando duas portas inversoras no CI HEF4049, elas foram utilizadas em paralelo com a readequação do sinal, evitando-se que portas ficassem sem utilização no circuito (o que poderia gerar ruído) e aumentando-se a capacidade de corrente do pulso readequado, o que evitou a necessidade de um driver extra para o acionamento do transistor.

Através do circuito apresentado na Figura 8, foi realizada a limitação da corrente de saída. A realimentação de corrente é feita através de uma liga de constantan inserida entre a referência da carga e a referência do circuito. A tensão presente na liga de constantan é amplificada e comparada com uma tensão fixa. Esta tensão deve ser ajustada de acordo com o valor de corrente que se deseja limitar (no caso do protótipo montado o valor foi de $150 \mathrm{~mA}$ ).

Figura 8 - Realimentação de tensão e corrente para a limitação da corrente de saída.

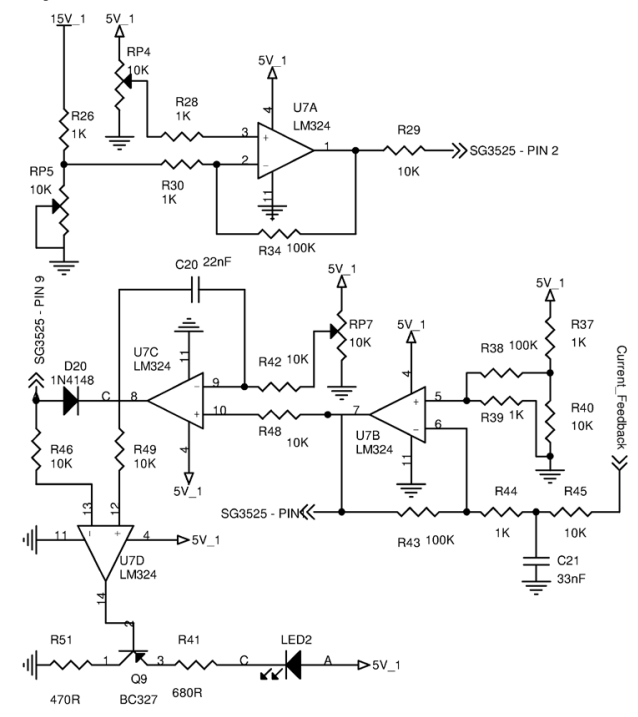

Fonte: Dados do autor
A saída do circuito limitador pode, através de um diodo, reduzir a tensão de comparação do circuito de controle, o que faz com que a tensão de saída caia, mantendo a corrente máxima. Para que a corrente máxima seja respeitada mesmo em uma situação de curto-circuito, a tensão de comparação deve ser igual à referência do circuito de controle, assim, fez-se necessário o deslocamento da referência do circuito de controle, visto que a mínima tensão possível no controle da corrente é a tensão de condução do diodo (por volta de $0,7 \mathrm{~V}$ ). Este deslocamento foi feito adicionando um diodo (D19 do Anexo 1) entre a referência do circuito de controle e a referência do circuito.

Outro ponto importante é a dupla alimentação do circuito de controle. Este circuito é alimentado inicialmente através da tensão não regulada, logo que a fonte passa a gerar uma tensão regulada, ela é utilizada para alimentar o circuito de controle, tornando-o imune as variações da tensão de entrada. Como o controle é alimentado, em certos momentos, através da tensão regulada por ele mesmo, é importante lembrar de somar o consumo do mesmo ao consumo desejado para a carga, na hora de projetar a fonte. Para o protótipo montado a tensão de saída foi especificada para $15 \mathrm{~V}$, a carga poderia consumir até $150 \mathrm{~mA}$, o circuito de controle consome 30mA nesta tensão (chaveando a $150 \mathrm{kHz}$ ) assim a fonte foi projetada para $15 \mathrm{~V} / 180 \mathrm{~mA}$. Esta informação é muito importante para o cálculo do rendimento das fontes secundárias.

\section{Resultados}

Visando aumentar a flexibilidade do sistema, optou-se por trabalhar com placas de circuito impresso diferentes para a fonte principal e para os pares de fontes secundárias. Isto permite a montagem de sistemas com mais ou menos fontes secundárias do que as 9 previstas inicialmente neste trabalho.

Enquanto o layout da placa das fontes secundárias estava sendo feito, observou-se que 
estas placas estavam assumindo tamanhos maiores que o previsto. Para tentar solucionar este problema separou-se esta placa em duas, a primeira ficou com a parte de potência e é utilizada como base para a segunda, que contém a parte de controle e deve ser soldada perpendicularmente a placa de potência através de uma barra de pinos de $90^{\circ}$ com 7 vias.

A placa que contém a parte de potência das fontes secundárias ficou com 96x30 mm e pode ser vista na Figura 9. É importante observar que o circuito do transformador isolador foi montando nesta placa.

Para reduzir o tamanho da placa que contém a parte de controle da fonte secundária optou-se por trabalhar com a maioria dos componentes no formato SMD. Esta placa ficou com 47x27 mm e pode ser vista na Figura 10.

A placa de controle ficou com dimensões bem reduzidas e como possui um circuito de controle genérico pode ser facilmente utilizada em outros projetos.

Figura 9 - Placa da parte de potência das fontes secundárias

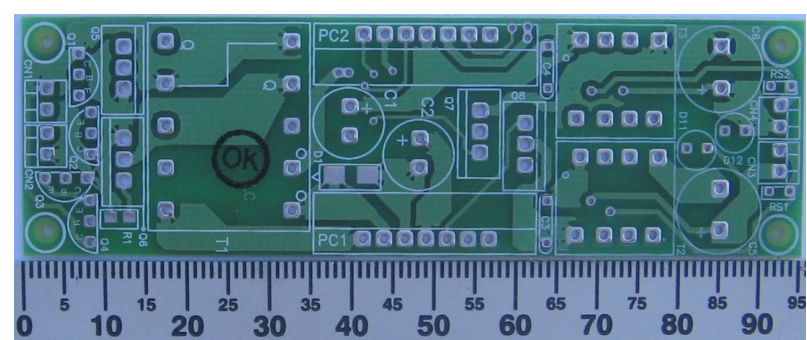

Fonte: Dados do autor

Figura 10 - Parte de Controle das fontes secundárias.

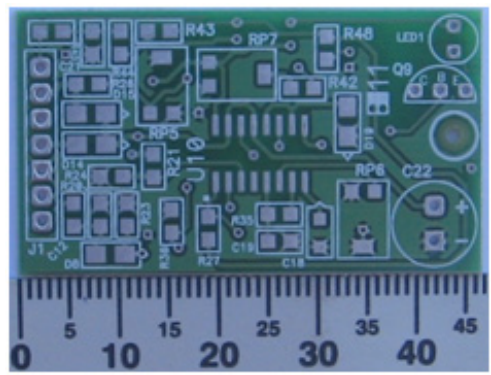

Fonte: Dados do autor
$\mathrm{Na}$ Figura 11 está apresentado um conjunto com um transformador isolador e duas fontes secundárias completas.

Figura 11 - Conjunto para duas saídas isoladas/ reguladas/sincronizadas.

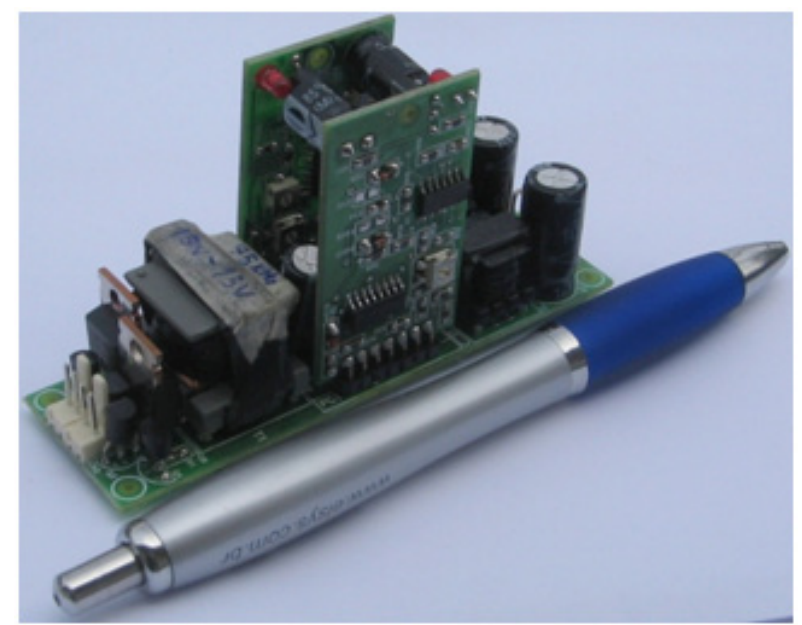

Fonte: Dados do autor

Com um osciloscópio digital de $60 \mathrm{MHz}$ e 1 GS/s (marca: Tektronix; modelo: TDS 2002) fez-se a aquisição dos sinais mais importantes do projeto, conforme figuras a seguir.

Na Figura 12 pode-se observar o sinal de entrada e as saídas do flip-flop, onde o sinal de entrada aparece divido por 2 e por 4 respectivamente.

Figura 12 - Saídas do flip-flop (Tensão: 10V/div. Tempo 2,5us/div).

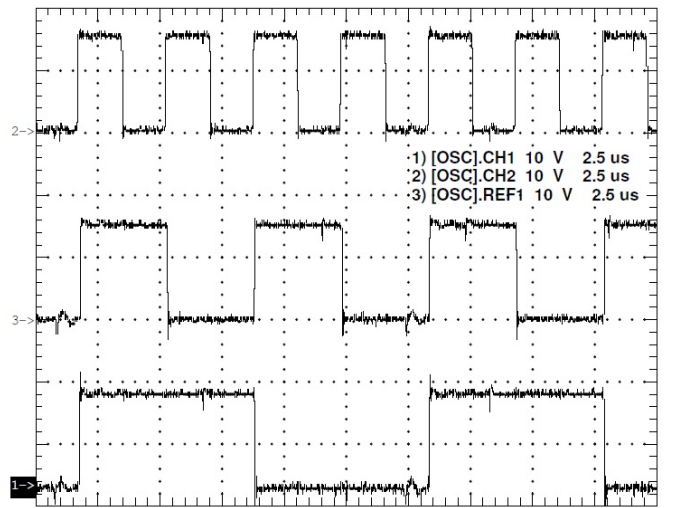

Fonte: Dados do autor 
$\mathrm{Na}$ Figura 13 está apresentado o tratamento empregado nos pulsos complementares para diminuir a dissipação nos transistores dos transformadores isoladores.

Figura 13 - Pulsos de acionamento alterados (Tensão: 10V/div. Tempo 5us/div).

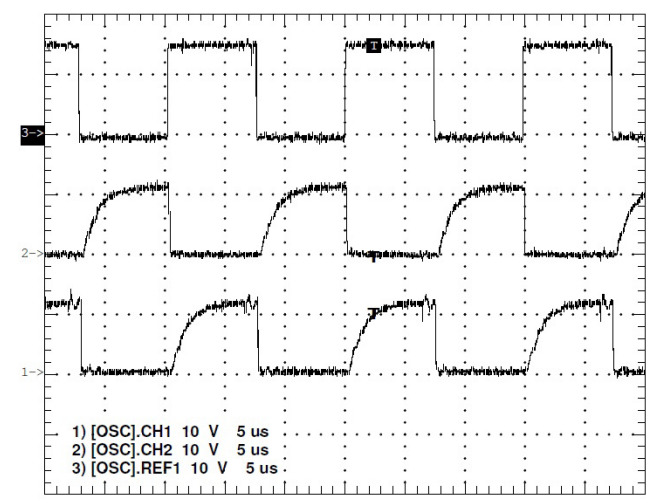

Fonte: Dados do autor

A Figura 14 mostra os pulsos gerados para o sincronismo da fonte principal e de uma das fontes secundárias (pulsos com duração aproximada de 800ns). Já a Figura 15 mostra o sincronismo entre o sinal externo e os pulsos da chave da fonte principal e da chave de uma das fontes secundárias. A fonte principal é sincronizada a um quarto da freqüência do sinal externo e as fontes secundárias são sincronizadas, devido à soma dos sinais de saída conforme mencionado anteriormente, com metade da freqüência do sinal externo.

Figura 14 - Pulsos de sincronismo (Tensão: 10V/ div. Tempo: 5 us/div).

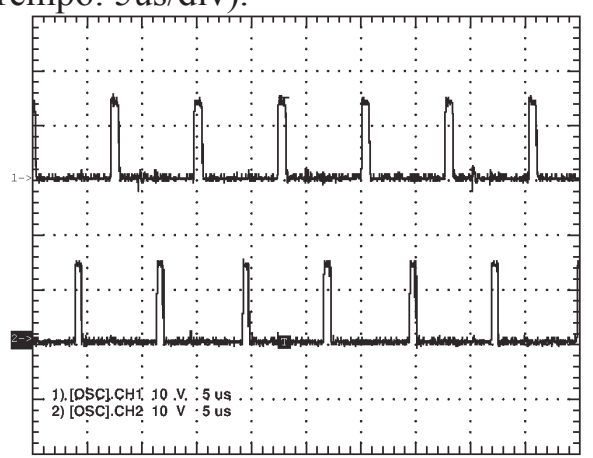

Fonte: Dados do autor
Figura 15 - Sincronismo entre o sinal externo, o chaveamento das fontes secundárias e da fonte principal (Tensão: 10V/div. Tempo 5us/div).

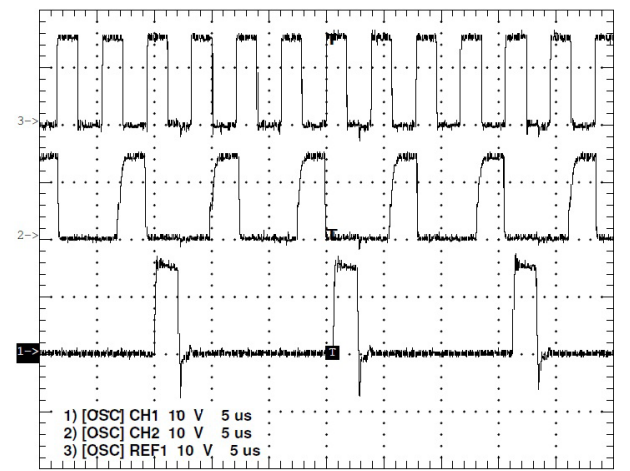

Fonte: Dados do autor

A Figura 16 traz a saída do circuito detector da presença do sinal externo no momento em que este sinal é inserido no sistema e no momento em que é retirado do sistema.

Figura 16 - Detecção do sinal externo (Tensão: 10V/div. Tempo 2,5us/div).

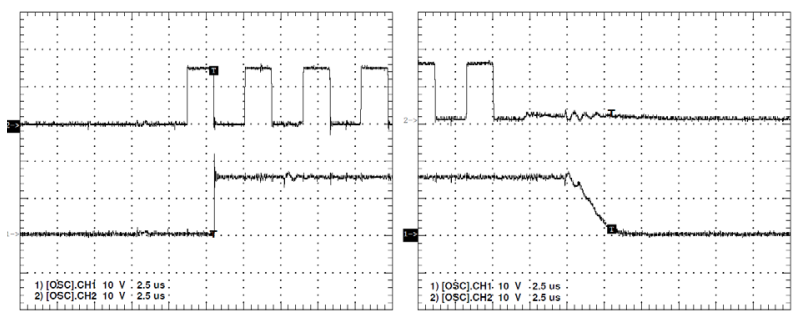

Fonte: Dados do autor

Quando o sinal externo não está presente, as fontes secundárias são automaticamente sincronizadas com a fonte principal. Este sincronismo está apresentado na Figura 17, através dos pulsos das chaves. 
Figura 17 - Auto sincronismo (Tensão: 10V/div. Tempo 5us/div).

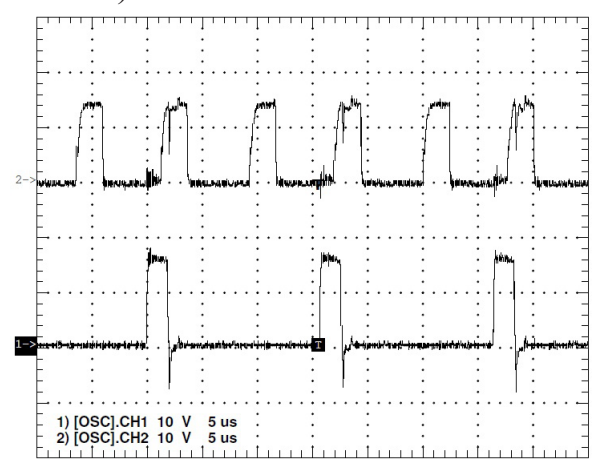

Fonte: Dados do autor

Na Figura 18 está presente a tensão de saída de uma das fontes secundárias (15V isolado, regulado, sincronizado).

O circuito SG3525 é recomendado para aplicações de maior potência, pois seu próprio consumo (próximo a $0,5 \mathrm{~W}$ ) pode diminuir o rendimento da fonte em questão, visto que a potência de saída das mesmas é de 2,25W. Outros fatores que diminuíram o rendimento das fontes secundárias foram a própria isolação (perdas nos transformadores isoladores) e o consumo do circuito limitador de corrente

Figura 18 - Tensão de saída (Tensão: 5V/div. Tempo 5us/div).

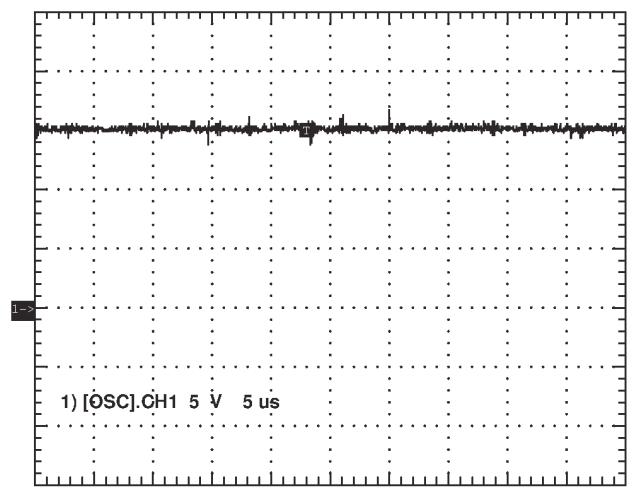

Fonte: Dados do autor
Na prática para 300mA na saída de um conjunto (150mA em cada fonte isolada) são consumidos $560 \mathrm{~mA}$ da fonte principal, um rendimento de 0,53 , porém, mesmo com este rendimento, seu peso, tamanho e volume compensam se comparado a tecnologia linear.

O consumo dos circuitos de controle com a isolação é de $120 \mathrm{~mA}$ para um conjunto, e se consideramos que estes circuitos são alimentados pela própria fonte secundária $\mathrm{o}$ rendimento real da mesma passa a ser de 0,75 ( [300mA + $120 \mathrm{~mA}] / 560 \mathrm{~mA})$. Assim, se o consumo do circuito de controle diminuir o rendimento aparente da fonte se aproximará dos $75 \%$.

Outro fator que influenciou o rendimento das fontes secundárias foi a escolha do núcleo utilizado. Embora os cálculos tenham fornecido o tamanho mínimo do núcleo, quando se trabalhou com um núcleo maior obteve-se um melhor rendimento, mas considerou-se que o tamanho final do sistema era mais importante que a diferença entre o rendimento dos núcleos, assim, optou-se por trabalhar com o menor núcleo possível.

\section{Comparação}

A título de comparação, considerou-se o circuito apresentado na Figura 19 (responsável por gerar as fontes isoladas em (FERREIRA, 2009)) como exemplar padrão da tecnologia linear, mais utilizada atualmente.

O núcleo marcado como "1" na Figura 19 é responsável por gerar a tensão isolada utiliza nas chaves da parte inferior do inversor e da alimentação do circuito de controle (já que em (FERREIRA, 2009) não se utilizou tensões negativas no desacionamente das chaves). Por isto esta parte do circuito será desconsiderada nesta comparação. 
O núcleo marcado como "4" na Figura 19 é responsável por gerar a tensão isolada para o circuito comutador de (FERREIRA, 2009) e por isto também será desconsiderado nesta comparação.

Os núcleos marcados como "2" e "3" na Figura 19 , bem como a parte da placa de circuito impresso logo abaixo dos mesmos, são responsáveis por gerar duas fontes de tensão isoladas e reguladas necessárias para o inversor do UPS, por isto serão comparados com a parte do sistema proposto responsável por gerar duas fontes isoladas e reguladas.

Figura 19: Circuito gerador de fontes isoladas de forma linear.

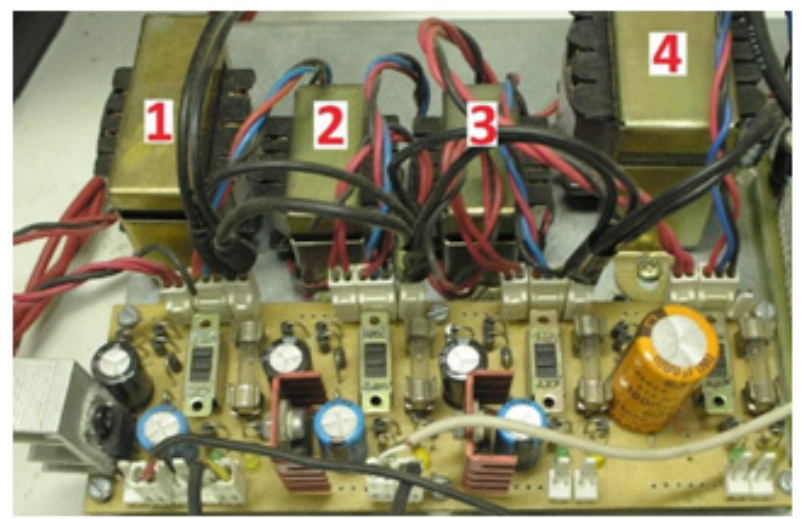

Fonte: FERREIRA, 2009

Para gerar as duas fontes isoladas, o circuito da Figura 19 utiliza dois transformadores $110 \mathrm{~V} / 220 \mathrm{~V}$ para $9 \mathrm{~V}+9 \mathrm{~V}$ de $200 \mathrm{~mA}$.

Todo o circuito utilizado para gerar as duas fontes isoladas e reguladas de maneira linear ocupou um espaço de 7,6cm de largura e $10,5 \mathrm{~cm}$ de comprimento, totalizando uma área de 79,8 cm2. O sistema proposto, de acordo com a Figura 11, para gerar as mesmas duas fontes isoladas e reguladas ocupa um espaço de $3 \mathrm{~cm}$ de largura por $9,6 \mathrm{~cm}$ de comprimento, totalizando uma área de $28,8 \mathrm{~cm} 2$. Logo, o sistema proposto ocupa apenas $36,1 \%$ da área que seria utilizada na tecnologia linear.

Embora a altura máxima do sistema proposto seja maior que o da tecnologia linear $(4,7 \mathrm{~cm}$ contra
$3,2 \mathrm{~cm}$ ) esta altura é mantida apenas em $2,6 \mathrm{~cm}$ da placa, assim, o volume total do sistema proposto também é menor.

Em relação ao peso, apenas os dois transformadores lineares chegam a $240 \mathrm{~g}$, enquanto toda a parte que gera as duas fontes isoladas do sistema proposto pesa $75 \mathrm{~g}$, logo o mesmo pesa apenas $31,25 \%$ do peso do sistema linear.

O rendimento do sistema proposto é de $53 \%$, bem abaixo do esperado para uma fonte chaveada, mas o rendimento típico de uma fonte linear fica entre $25 \%$ e $50 \%$ (POMILIO, 2011) logo o sistema proposto não fica em desvantagem neste quesito.

\section{Conclusões}

Como foi demonstrado no decorrer deste trabalho a geração de fontes isoladas, reguladas e sincronizadas é de extrema importância para aplicações em UPS (e muitos outros circuitos que necessitem de referências distintas), mesmo assim este assunto não é abordado na maioria dos trabalhos apresentados sobre o tema. Assim, este trabalho pode servir como base para futuros projetos de UPS.

As formas de isolação, sincronismo e controle de corrente apresentadas, demonstraram-se muito eficazes e de fácil adequação para diferentes valores de tensão, carga e freqüência, sendo recomendadas para novos projetos.

O circuito de controle utilizado mostrou-se pouco eficiente para este nível de potência, reduzindo drasticamente o rendimento do projeto, assim, para trabalhos futuros serão buscadas novas formas para o controle das fontes de baixa potência, bem como pode ser estudado a utilização de núcleos de ferrite com faixa de histerese estreita da curva $\mathrm{BxH}$, diodos do tipo Schottky para diminuir a tensão direta, capacitores com baixa resistência série equivalente e transistores mosfets mais rápidos.

Com relação ao tamanho, peso e volume o sistema proposto demonstrou ser muito menor que o da tecnologia mais utilizada atualmente. 
Se considerarmos apenas as nove fontes isoladas (desconsiderando a fonte principal de ambas as tecnologias) o sistema proposto gera uma economia de $229,5 \mathrm{~cm} 2$ na área ocupada e $742,5 \mathrm{~g}$ no peso do mesmo, o que é muito significativo.

\section{Referências}

AGÊNCIA NACIONAL DE ENERGIA ELÉTRICA. ANEEL. Falta de energia gera $R \$ 152$ milhões em compensação até junho de 2010. 2011. Disponível em: $<$ http://www.aneel.gov.br/aplicacoes/noticias/Output Noticias.cfm?Identidade $=3824 \&$ id area $=90>$. Acesso em: 6 jun. 2011.

CÂMARA, R. A.; CRUZ, C. M. T.; BASCOPÉ, R. P. T. Retificador Boost Baseado Na Célula De Comutação De Três Estados Para Aplicações Em Nobreaks. Revista Brasileira de Eletrônica de Potência (SOBRAEP), Campinas, v. 15, n. 4, p. 275-283, 2010.

CANÔNICO, R. B. Amplificador de áudio tipo classe D, resposta em frequência $20 \mathrm{~Hz}$ a $20 \mathrm{kHz}$, alta fidelidade e modulação multiplexada. 2011. Dissertação (Mestrado Engenharia Elétrica) - Universidade Estadual de Londrina, Londrina.

FERREIRA, A. L. B. UPS de $5 \mathrm{kV}$ A, tipo Passive Standby, com integração de painéis solares. 2009. Dissertação. (Mestrado em Engenharia Eletrica) - Universidade Estadual de Londrina, Londrina.

KASTER, M. S.; PAGANO, D. J. Geração de oscilações auto-sustentadas em inversores monofásicos. Revista Controle \& Automação, Campinas, v. 17, n. 1, p. 61-70, 2006.

LINARD, F. M. A.; CRUZ, C. M. T.; BASCOPÉ, R. P. T.; OLIVEIRA JÚNIOR, D. S. Sistema ininterrupto de energia de dupla conversão com integração do retificador e inversor. In: CONFERÊNCIA INTERNACIONAL DE APLICAÇÕES INDUSTRIAIS, 8., 2008, Poços de Caldas. Anais... Poços de Caldas: INDUSCON, 2008.

MELLO, L. F. P. Análise e projeto de fontes chaveadas. São Paulo: Érica, 1996.

MILNE, G. W.; JANSEN, E.; ROUX, J. J.; KOEKEMOER, J. A. EMC and RFI problems and solutions on the SUNSAT micro-satellite. In: SOUTH AFRICAN SYMPOSIUM ON COMMUNICATIONS AND SIGNAL PROCESSING, 1998, South Africa. Proceedings... South África: Stellenbosch University, 1998. v. 1, n. 98EX214, p. 293-298.

POMILIO, J. A. EE833 eletrônica de potência: módulo 5. Campinas: UNICAMP/FEE, 2011.
SCHIAVON, G. J. No-Break 1,2KVA, senoidal, operando em malha fechada: circuito de potência, circuito de controle analógico e circuito de controle digital com DSC. 2007. Dissertação (Mestrado Engenharia Elétrica) - Universidade Estadual de Londrina, Londrina.

SCHUCH, L.; RECH, C.; PINHEIRO, H.; HEY, H. L.; GRÜNDLING, H. A.; PINHEIRO, J. R. Análise e Projeto de um Conversor Bidirecional PWM de Alto Desempenho para Interface entre o Barramento CC e o Banco de Baterias. Revista Brasileira de Eletrônica de Potência (SOBRAEP), Campinas, v. 9, n. 1, p. 1-11, 2004.

TREVISO, C. H. G.; DEMIAN JUNIOR, A. E.; FERREIRA, A. L. B. No-break Para Aplicações Residenciais, Comerciais E Industriais de 5kw Com Interface Para Painel Solar (SOBRAEP). Revista Brasileira de Eletrônica de Potência (SOBRAEP), Campinas, v. 14, n. 3, p. 173-180, 2009.

TREVISO, C. H. G.; SCHIAVON, G. J. Complete Design For a $1.2 \mathrm{kVA}$ Uninterruptable Power Supply System With a Stabilized Sine Wave. In: CONGRESSO BRASILEIRO DE ELETRÔNICA DE POTÊNCIA, 9., 2007, Blumenau. Anais... Blumenau: COBEP, 2007. p. 1004-1009.

Recebido em 5 Outubro 2011- Received on October 5, 2011.

Aceito em 27 Dezembro, 2011 - Accepted on December 27, 2011. 


\section{ANEXO 1: CIRCUITO DE CONTROLE COMPLETO E CIRCUITO DE UMA DAS FONTES SECUNDÁRIAS}
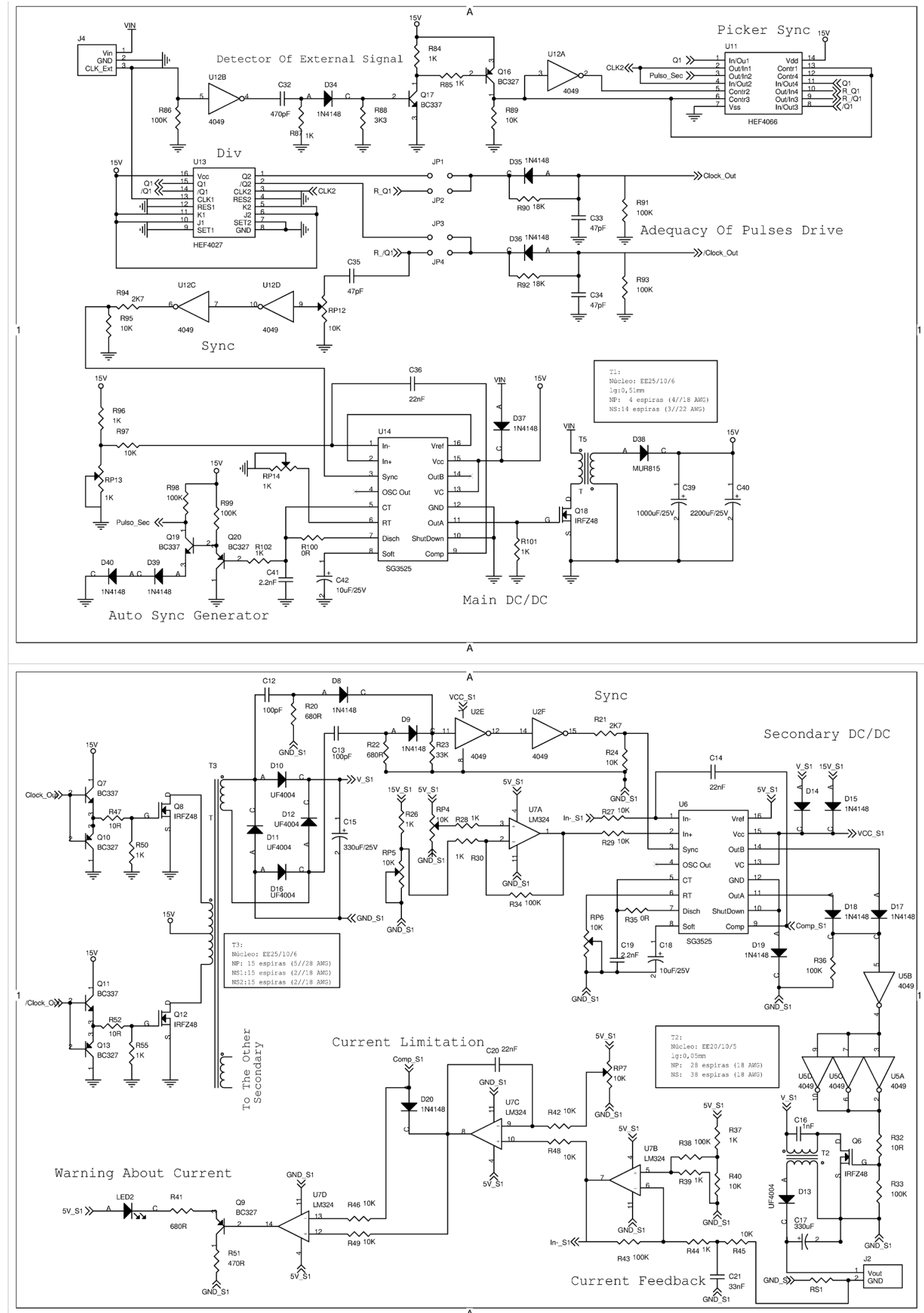